Research Article

\title{
Finite Time Inverse Optimal Stabilization for Stochastic Nonlinear Systems
}

\author{
Xiushan Cai, ${ }^{1}$ Yuhang Lin, ${ }^{1}$ and Wei Zhang ${ }^{2}$ \\ ${ }^{1}$ College of Mathematics, Physics, and Information Engineering, Zhejiang Normal University, Jinhua 321004, China \\ ${ }^{2}$ Laboratory of Intelligent Control and Robotics, Shanghai University of Engineering Science, Shanghai 201620, China \\ Correspondence should be addressed to Xiushan Cai; xiushancai@163.com
}

Received 30 April 2013; Accepted 20 June 2013

Academic Editor: Ljubisa Kocinac

Copyright (c) 2013 Xiushan Cai et al. This is an open access article distributed under the Creative Commons Attribution License, which permits unrestricted use, distribution, and reproduction in any medium, provided the original work is properly cited.

\begin{abstract}
This paper deals with finite time inverse optimal stabilization for stochastic nonlinear systems. A concept of the stochastic finite time control Lyapunov function (SFT-CLF) is presented, and a control law for finite time stabilization for the closed-loop system is obtained. Furthermore, a sufficient condition is developed for finite time inverse optimal stabilization in probability, and a control law is designed to ensure that the equilibrium of the closed-loop system is finite time inverse optimal stable. Finally, an example is given to illustrate the applications of theorems established in this paper.
\end{abstract}

\section{Introduction}

In many engineering fields, it is desirable that trajectories of a dynamical system converge to an equilibrium in finite time. In order to achieve convergence in finite time, one notices immediately that finite time differential equations cannot be Lipschitz at the origin. As all solutions reach zero in finite time, there is nonuniqueness of solutions through zero in backwards time. Haimo [1] pointed out that this violates the uniqueness condition for solutions of the Lipschitz differential equations. Finite time stability was studied in [13]. Recently, finite time stability has been further extended to switched systems in Orlov [4], time-delay systems in Moulay et al. [5], and impulsive dynamical systems in Nersesov et al. [6]. The problem of finite time stabilization has been studied by Bhat and Bernstein [7] and Hong et al. [8]. Finitetime stabilization technique has been applied in tracking control of multiagent systems by $\mathrm{Li}$ et al. [9] and attitude tracking control of spacecraft by Du et al. [10, 11]. Moulay and Perruquetti [12] studied finite time stabilization of a class of continuous system using the control Lyapunov functions (CLFs). The CLF was introduced by Artstein [13] and Sontag [14] and made a tremendous impact on stabilization theory. In particular Sontag's universal formula in [15] has played an important role in control theory. Florchinger [16] proved that the feedback control law defined in [15] can globally asymptotically stabilize stochastic nonlinear systems. This result was extended when the drift as well as the controlled part was corrupted by a noise in the works of Chabour and Oumoun [17].

After the success of finite time stability and stabilization theory for deterministic systems, how to extend them to the case of stochastic systems naturally became an important research area. Chen and Jiao [18-20] presented a new concept of finite time stability for stochastic nonlinear systems, and a theorem concerning the finite time stability was proved. However, to the authors knowledge, no work on finite time inverse optimal stabilization for stochastic systems has been done at the present stage.

In this paper, for general stochastic systems affine in the control and noise inputs, a concept of the stochastic finite time control Lyapunov function (SFT-CLF) is given. Next, a sufficient condition is developed for finite time stabilization in probability, and a control law is designed. After considering the finite time stabilization of stochastic systems, an important problem is how to further design a stabilizing controller which is also optimal with respect to meaningful cost functionals, that is, the inverse optimal control. In this paper, we consider the finite time inverse optimal controller design. This result is extended from the inverse optimality result of Freeman and Kokotovic [21] to finite time inverse 
optimal controller design for stochastic nonlinear systems. Finally, the effectiveness of the proposed design technique is illustrated by an example.

\section{System Description and Preliminaries}

Consider the following stochastic nonlinear system:

$$
d x=(f(x)+g(x) u) d t+h(x) d w,
$$

where $x \in R^{n}, u \in R^{m}$ are the state and the input of the system, respectively, $w$ is an $r$-dimensional independent standard Wiener process, and $f: R^{n} \rightarrow R^{n}, g: R^{n} \rightarrow R^{n \times m}$, and $h: R^{n} \rightarrow R^{n \times r}$ are continuous with $f(0)=0, h(0)=0$.

Let $V: R^{n} \rightarrow R^{+}$be a continuous function. $V$ is said to be positive definite if $V(0)=0$ and $V(x)>0$ for $x \neq 0$; $V$ is said to be radially unbounded if $V(x) \rightarrow \infty$ as $\|x\| \rightarrow \infty$.

For any given twice continuous differentiable function $V(x)$, associated with stochastic system (1), the infinitesimal generator $\mathscr{L}$ is defined as follows:

$$
\mathscr{L} V(x)=\frac{\partial V}{\partial x}(f(x)+g(x) u)+\frac{1}{2} \operatorname{Tr}\left\{h^{T}(x) \frac{\partial^{2} V}{\partial x^{2}} h(x)\right\} .
$$

In this paper, $K$ denotes the set of all functions $R^{+} \rightarrow$ $R^{+}$, which are continuous, strictly increasing, and vanishing at zero.

Definitions 1 and 2 and Lemma 3 given in $[19,20]$ and Lemma 4 given in [22] will be useful throughout this paper.

Definition 1. Assume that the system

$$
d x=f(x) d t+h(x) d w
$$

has the unique and global solution denoted by $x\left(t, x_{0}\right), 0 \leq$ $t<+\infty$, where $x_{0}$ is the initial state. Define $T_{0}\left(x_{0}, w\right)=$ $\inf \left\{T \geq 0: x\left(t, x_{0}\right)=0, \forall t \geq T\right\}$, which is called the stochastic settling time function. In particular, $T_{0}\left(x_{0}, w\right)=+\infty$ if $x\left(t, x_{0}\right) \neq 0$, for all $t \geq 0$.

Definition 2. For stochastic system (3), the equilibrium $x=0$ is said to be globally finite time stable in probability, if the following conditions hold:

(1) the equilibrium $x=0$ is globally stable in probability if for all $\varepsilon>0$ there exists a class $K$ function $\gamma(\cdot)$ such that $P\left\{\left\|x\left(t, x_{0}\right)\right\|<\gamma\left(\left\|x_{0}\right\|\right)\right\} \geq 1-\varepsilon$, for all $t \geq 0$, for all $x_{0} \in R^{n} \backslash\{0\}$,

(2) $E\left[T_{0}\left(x_{0}, w\right)\right]<\infty$, for any $x_{0} \in R^{n} \backslash\{0\}$.

Lemma 3. Assume that system (3) has the unique global solution in forward time for all initial conditions. If there exist a positive definite, twice continuous differentiable and radially unbounded Lyapunov function $V: R^{n} \rightarrow R^{+}$and a continuous differentiable, function $r: R^{+} \rightarrow R^{+}$such that

(i) $\mathscr{L} V(x) \leq-r(V(x))$,

(ii) for any $0 \leq \varepsilon<+\infty, \quad \int_{0}^{\varepsilon} \frac{1}{r(v)} d v<+\infty$,

( iii ) for $v>0, \quad r^{\prime}(v)>0$, then the equilibrium $x=0$ of system (3) is globally finite time stable in probability, and the settling time function $T_{0}\left(x_{0}, w\right)$ satisfies $E\left[T_{0}\left(x_{0}, w\right)\right] \leq \int_{0}^{V\left(x_{0}\right)}(1 / r(v)) d v$, which implies that $T_{0}\left(x_{0}, w\right)<+\infty$ almost surely (a.s.).

Lemma 4. Assume that

(i) $\|f(x)\| \leq c_{1}(1+\|x\|),\|h(x)\|^{2} \leq c_{1}\left(1+\|x\|^{2}\right)$ with $c_{1} \geq 0$,

(ii) $f(x)$ and $h(x)$ are continuous,

(iii) for each $N=1,2, \ldots$, it holds that $2\left\langle x-x^{\prime}, f(x)-\right.$ $\left.f\left(x^{\prime}\right)\right\rangle+\left\|h(x)-h\left(x^{\prime}\right)\right\|^{2} \leq c_{N} \rho_{N}\left(\left\|x-x^{\prime}\right\|^{2}\right)$, as $\|x\| \leq$ $N,\left\|x^{\prime}\right\| \leq N$, where $c_{N} \in R$, and $\rho_{N}(\sigma) \geq 0$, as $\sigma \geq 0$, is nonrandom, strictly increasing, continuous, and concave such that $\int_{0+} d u / \rho_{N}(u)=\infty$. Then for any given constant $x_{0} \in R^{n}$, (3) has a pathwise unique strong solution.

The stochastic finite time control Lyapunov function is defined as follows.

Definition 5. A positive definite, twice continuous differentiable, and radially unbounded function $V: R^{n} \rightarrow R^{+}$is a stochastic finite time control Lyapunov function (SFT-CLF) of system (1) if there exist real numbers $c>0$ and $0<\alpha<1$, such that

$$
\begin{aligned}
& \frac{\partial V}{\partial x} g(x)=0, \quad x \neq 0 \\
& \Longrightarrow \frac{\partial V}{\partial x} f(x)+\frac{1}{2} \operatorname{Tr}\left\{h^{T}(x) \frac{\partial^{2} V}{\partial x^{2}} h(x)\right\} \leq-c(V(x))^{\alpha}
\end{aligned}
$$

$V(x)$ is said to satisfy small control property with respect to system (1) if for each $\varepsilon>0$ there is a $\sigma>0$ such that, for all $x \neq 0$ satisfying $\|x\|<\sigma$, there is some $u$ with $\|u\|<\varepsilon$ such that $(\partial V / \partial x) f(x)+(\partial V / \partial x) g(x) u+(1 /$ 2) $\operatorname{Tr}\left\{h^{T}(x)\left(\partial^{2} V / \partial x^{2}\right) h(x)\right\} \leq-c(V(x))^{\alpha}, c>0,0<\alpha<1$.

\section{Main Results}

Consider system (1). An explicit feedback control law is designed such that the equilibrium $x=0$ of the closed-loop system is globally finite time stable in probability. Moreover, a control law for finite time inverse optimal stabilization is dealt with.

\subsection{Finite Time Stabilization in Probability}

Theorem 6. Consider system (1). If $V(x)$ is an SFT-CLF for system (1) such that the following inequality holds

$$
\lim _{\delta \rightarrow 0} \sup _{x \in N(\delta)} \frac{a(x)}{\|b(x)\|} \leq 0
$$


with

$$
\begin{gathered}
a(x)=\frac{\partial V}{\partial x} f(x)+\frac{1}{2} \operatorname{Tr}\left\{h^{T}(x) \frac{\partial^{2} V}{\partial x^{2}} h(x)\right\}+c(V(x))^{\alpha}, \\
c>0,0<\alpha<1, \\
b(x)=\frac{\partial V}{\partial x} g(x), \\
N(\delta)=\left\{x \in R^{n} \mid 0<\|x\| \leq \delta\right\},
\end{gathered}
$$

let the control law be

$$
\begin{aligned}
u & =k(x) \\
& = \begin{cases}-b^{T}(x) \frac{a(x)+\sqrt{a^{2}(x)+\|b(x)\|^{4}}}{\|b(x)\|^{2}}, & b(x) \neq 0, \\
0, & b(x)=0 .\end{cases}
\end{aligned}
$$

In addition, assume that the control law (8) is such that the closed-loop system has the unique global solution in forward time for all initial conditions. Then the equilibrium $x=0$ of the closed-loop system (1) and (8) is globally finite time stable in probability. And the settling time function $T_{0}\left(x_{0}, w\right)$ satisfies $E\left[T_{0}\left(x_{0}, w\right)\right] \leq\left(V\left(x_{0}\right)\right)^{1-\alpha} / c(1-\alpha)$.

Proof. Take $r(v)=c v^{\alpha}$, with $c>0$ and $0<\alpha<1$. Since $V(x)$ is an SFT-CLF for system (1), it implies that

$$
\begin{aligned}
& \frac{\partial V}{\partial x} g(x)=0, \quad x \neq 0 \\
& \Longrightarrow \frac{\partial V}{\partial x} f(x)+\frac{1}{2} \operatorname{Tr}\left\{h^{T}(x) \frac{\partial^{2} V}{\partial x^{2}} h(x)\right\} \leq-r(V(x)) .
\end{aligned}
$$

Consider system (1). We have $\mathscr{L} V(x)=(\partial V / \partial x)(f(x)+$ $g(x) u)+(1 / 2) \operatorname{Tr}\left\{h^{T}(x)\left(\partial^{2} V / \partial x^{2}\right) h(x)\right\}$. If $b(x) \neq 0$ and by $(8)$, it can be deduced that

$$
\begin{aligned}
\mathscr{L} V(x)= & \frac{\partial V}{\partial x} f(x)-a(x)-\sqrt{a^{2}(x)+\|b(x)\|^{4}} \\
& +\frac{1}{2} \operatorname{Tr}\left\{h^{T}(x) \frac{\partial^{2} V}{\partial x^{2}} h(x)\right\} \\
& =-c(V(x))^{\alpha}-\sqrt{a^{2}(x)+\|b(x)\|^{4}} \\
& <-c(V(x))^{\alpha} \\
& =-r(V(x)) .
\end{aligned}
$$

If $b(x)=0, x \neq 0$, in view of (8) and (9), it yields

$$
\mathscr{L} V(x)=\frac{\partial V}{\partial x} f(x)+\frac{1}{2} \operatorname{Tr}\left\{h^{T}(x) \frac{\partial^{2} V}{\partial x^{2}} h(x)\right\} \leq-r(V(x)),
$$

when $b(x)=0, x \neq 0$. So $\mathscr{L} V(x) \leq-r(V(x))$; that is, condition (i) of Lemma 3 holds. It is also easy to verify that conditions (ii) and (iii) in Lemma 3 hold.
We will prove that the control law $u=k(x)$ given by (8) is differentiable away from the origin and continuous at the origin. For this objective, consider an open subset of $R^{2}$ as follows:

$$
S=\left\{(\zeta, \eta) \in R^{2} \mid \eta \neq 0 \text { or } \zeta<0\right\} .
$$

From implicit function existence theorem, the function defined by

$$
\phi(\zeta, \eta)= \begin{cases}\frac{\zeta+\sqrt{\zeta^{2}+\eta^{2}}}{\eta}, & \eta \neq 0, \\ 0, & \eta=0\end{cases}
$$

is differentiable on $S$. By (9), for any $x \in R^{n} \backslash\{0\}$, we have $\left(a(x),\|b(x)\|^{2}\right) \in S$. So the control law (8) is differentiable away from the origin. If (6) holds, it implies that the control law (8) satisfies the small control property by [23]. Then it is continuous at the origin by [15].

In addition, under the control law (8), the equilibrium $x=$ 0 of the closed-loop system has the unique global solution in forward time. From Lemma 3, the closed-loop system (1) and (8) is globally finite time stable in probability. And the settling time function $T_{0}\left(x_{0}, w\right)$ satisfies $E\left[T_{0}\left(x_{0}, w\right)\right] \leq$ $\int_{0}^{V\left(x_{0}\right)}(1 / r(v)) d v=\left(V\left(x_{0}\right)\right)^{1-\alpha} / c(1-\alpha)$.

3.2. Finite Time Inverse Optimal Stabilization in Probability. In this subsection, we consider finite time inverse optimal stabilization in probability. That is, a feedback control law $u(x)$ for system (1) will be constructed such that the following properties hold:

(i) the closed-loop system is finite time stable in probability at the equilibrium $x=0$,

(ii) $u(x)$ minimizes the cost functional

$$
J\left(u, x, x_{0}, w\right)=E\left\{\int_{0}^{T_{0}\left(x_{0}, w\right)}\left(l(x)+u^{T} R(x) u\right) d t\right\},
$$

where $l(x) \geq 0, R(x)>0$ for all $x$ and $T_{0}\left(x_{0}, w\right)$ is the settlingtime function, and $x_{0} \in R^{n}$ is an initial value.

In the inverse approach, a finite time stabilizing feedback law $u(x)$ is designed first, and then it is shown that the feedback law is to find $l(x) \geq 0$ and $R(x)>0$ such that $u(x)$ optimizes (14). The problem is inverse because the functions $l(x)$ and $R(x)$ are a posteriori determined by the stabilizing feedback law, rather than a priori chosen by the designer.

Theorem 7. Consider system (1). If $V(x)$ is an SFT-CLF for system (1) such that (6) holds, then let the control law be

$$
\begin{aligned}
& u^{*} \\
& = \begin{cases}-b^{T}(x)\left(\lambda+\frac{a(x)+\sqrt{a^{2}(x)+\|b(x)\|^{4}}}{\|b(x)\|^{2}}\right), & b(x) \neq 0, \\
0, & b(x)=0,\end{cases}
\end{aligned}
$$


where $\lambda>0$ and $a(x), b(x)$ are defined as (7). In addition, assume that the control law (15) is such that the closed-loop system has the unique global solution in forward time for all initial conditions. Then the control law (15) solves the problem of finite time inverse optimal stabilization in probability for system (1) by minimizing the cost functional (14) with

$$
\begin{aligned}
& R^{-1}(x)= \begin{cases}2 \lambda+2 \frac{a(x)+\sqrt{a^{2}(x)+\|b(x)\|^{4}}}{\|b(x)\|^{2}}, & b(x) \neq 0, \\
2 \lambda, & b(x)=0\end{cases} \\
& l(x)=-\frac{\partial V}{\partial x} f(x)-\frac{1}{2} \operatorname{Tr}\left\{h^{T}(x) \frac{\partial^{2} V}{\partial x^{2}} h(x)\right\} \\
& \quad+\frac{1}{4} R^{-1}(x)\left\|\frac{\partial V}{\partial x} g(x)\right\|^{2} .
\end{aligned}
$$

And the settling time function $T_{0}\left(x_{0}, w\right)$ satisfies $E\left[T_{0}\left(x_{0}\right.\right.$, $w)] \leq\left(V\left(x_{0}\right)\right)^{1-\alpha} / c(1-\alpha)$.

Proof. It is easy to prove that $R(x)>0$. Next, we will prove that $l(x) \geq 0$.

Substituting $R^{-1}(x)$ into $l(x)$, we have

$$
\begin{aligned}
l(x)= & -\frac{\partial V}{\partial x} f(x)-\frac{1}{2} \operatorname{Tr}\left\{h^{T}(x) \frac{\partial^{2} V}{\partial x^{2}} h(x)\right\} \\
& +\frac{1}{4} R^{-1}(x)\left\|\frac{\partial V}{\partial x} g(x)\right\|^{2} \\
= & \frac{1}{2}\left(\sqrt{a^{2}(x)+\|b(x)\|^{4}}-a(x)\right) \\
& +c(V(x))^{\alpha}+\frac{1}{2} \lambda\|b(x)\|^{2}
\end{aligned}
$$$$
>0 \text {, }
$$

when $b(x) \neq 0$; since $V(x)$ is an STF-CLF for system (1), it yields

$$
\begin{aligned}
l(x) & =-\frac{\partial V}{\partial x} f(x)-\frac{1}{2} \operatorname{Tr}\left\{h^{T}(x) \frac{\partial^{2} V}{\partial x^{2}} h(x)\right\} \\
& \geq c(V(x))^{\alpha} \geq 0,
\end{aligned}
$$

when $b(x)=0, x \neq 0$. In addition, it is easy to obtain $l(x)=$ 0 if $x=0$. In conclusion, it yields $l(x) \geq 0$. Thus the cost functional (14) with $l(x)$ and $R(x)$ given by (16) is meaningful.

Consider the closed-loop system (1) and (15). Since $V(x)$ is an SFT-CLF for system (1), we get that

$$
\begin{aligned}
\mathscr{L} V(x) & =\frac{\partial V}{\partial x}\left(f(x)+g(x) u^{*}\right)+\frac{1}{2} \operatorname{Tr}\left\{h^{T}(x) \frac{\partial^{2} V}{\partial x^{2}} h(x)\right\} \\
& =-\lambda\|b(x)\|^{2}-c(V(x))^{\alpha}-\sqrt{a^{2}(x)+\|b(x)\|^{4}} \\
& <-c(V(x))^{\alpha},
\end{aligned}
$$

whenever $b(x) \neq 0$ and $\mathscr{L} V(x)=(\partial V / \partial x) f(x)+(1 /$ 2) $\operatorname{Tr}\left\{h^{T}(x)\left(\partial^{2} V / \partial x^{2}\right) h(x)\right\} \leq-c(V(x))^{\alpha}$ otherwise. Since (6) holds, it can be deduced that the control law (15) is continuous on $R^{n}$. In addition, the control law (15) is such that the closed-loop system has the unique global solution in forward time. Thus $u^{*}$ is such that the equilibrium $x=0$ of the closedloop system (1) and (15) is globally finite time stable in probability, and the settling time function $T_{0}\left(x_{0}, w\right)$ satisfies $E\left[T_{0}\left(x_{0}, w\right)\right] \leq\left(V\left(x_{0}\right)\right)^{1-\alpha} / c(1-\alpha)$, which implies $T_{0}\left(x_{0}\right.$, $w)<+\infty$ a.s.

Let $x\left(t, x_{0}\right)$ be the unique and global solution of the closed-loop system (1) and (15) from the initial value $x_{0}$. Since the equilibrium $x=0$ of the closed-loop system (1) and (15) is globally finite time stable in probability, it implies that $x\left(t, x_{0}\right)=0, t \geq 0$ when $x_{0}=0$. Consider $x_{0} \neq 0$, there must exist $k \in N=:\{1,2,3, \ldots\}$ such that $1 / k<\left|x_{0}\right|<k$. Define an increasing stop time sequence as follows:

$$
\tau_{k}=\inf \left\{t \geq 0:\left|x\left(t, x_{0}\right)\right| \notin\left(\frac{1}{k}, k\right)\right\},
$$

recalling that the Itô differential of $V$ is

$$
d V=\mathscr{L} V(x) d t+\frac{\partial V}{\partial x} h(x) d w
$$

Since $(\partial V(x(t)) / \partial x) h(x(t))$ is bounded on $\left[0, \tau_{k} \wedge k\right]$, it yields

$$
\int_{0}^{\tau_{k} \wedge k}\left(\frac{\partial V(x(s))}{\partial x} h(x(s))\right)^{2} d s<\infty .
$$

According to the property of Itô's integral [24, page 143], we get

$$
E\left\{\int_{0}^{\tau_{k} \wedge k} \frac{\partial V(x(s))}{\partial x} h(x(s)) d w\right\}=0
$$

And in view of (21), one can deduce that

$$
E\left\{V\left(x_{0}\right)-V\left(x\left(\tau_{k} \wedge k\right)\right)+\int_{0}^{\tau_{k} \wedge k} \mathscr{L} V(x(\tau)) d \tau\right\}=0 .
$$

Since $x\left(t, x_{0}\right)$ is unique and global solution, noticing Definition 1 , let $k \rightarrow \infty$. We get $\tau_{k} \wedge k \rightarrow T_{0}\left(x_{0}, w\right)$ a.s. So it yields

$$
\begin{aligned}
E & \int_{0}^{T_{0}\left(x_{0}, w\right)} \mathscr{L} V(x(\tau)) d \tau \\
& =\lim _{t \rightarrow T_{0}^{-}\left(x_{0}, w\right)} E(V(x(t)))-E\left(V\left(x_{0}\right)\right) .
\end{aligned}
$$

Next, we prove that $\lim _{t \rightarrow T_{0}^{-}\left(x_{0}, w\right)} E(V(x(t)))=0$.

Denote $T_{0}=T_{0}\left(x_{0}, w\right)$. Noting that $T_{0}<+\infty$ a.s. and by the continuity of the solution, it holds that

$$
\lim _{t \rightarrow T_{0}^{-}} x(t)=\lim _{t \rightarrow 0^{-}} x\left(T_{0}-t\right)=\lim _{n \rightarrow \infty} x\left(T_{0}-\frac{T_{0}}{n}\right)=\lim _{n \rightarrow \infty} x_{n},
$$

where $x_{n}=x\left(T_{0}-T_{0} / n\right)$. 
Since $\lim _{n \rightarrow \infty} x_{n}=0$ a.s., we have

$$
\lim _{n \rightarrow \infty} V\left(x_{n}\right)=0 \text { a.s. }
$$

Denote $\varphi_{n}=E\left(V\left(x_{n}\right)\right)$, and in view of $\mathscr{L} V(x) \leq$ $-c(V(x))^{\alpha}$, then $\varphi_{n}$ is monotone decreasing sequence. So there exists a constant $\varphi$ such that $\lim _{n \rightarrow \infty} E\left(V\left(x_{n}\right)\right)=\varphi$. If $\varphi \neq 0$, then there exists a convergent subsequence $\left\{x_{n^{\prime}}\right\}$ such that $\lim _{n^{\prime} \rightarrow \infty} V\left(x_{n^{\prime}}\right)=\varphi \neq 0$, a.s. However, this contradicts the conclusion (27), so $\lim _{n \rightarrow \infty} E\left(V\left(x_{n}\right)\right)=0$. By the continuity of the solution, we know that

$$
\lim _{t \rightarrow T_{0}^{-}} E(V(x(t)))=\lim _{n \rightarrow \infty} E\left(V\left(x_{n}\right)\right)=0 .
$$

Finally, we prove optimality. Substituting $l(x)$ and $u=v-$ $(1 / 2) R^{-1}(x) b^{T}(x)$ into $J\left(u, x, x_{0}, w\right)$, we have

$$
\begin{aligned}
& J\left(u, x, x_{0}, w\right) \\
& =E\left\{\int_{0}^{T_{0}}\left(l(x)+u^{T} R(x) u\right) d t\right\} \\
& =E\left\{\int _ { 0 } ^ { T _ { 0 } } \left(-\frac{\partial V}{\partial x} f(x)-\frac{1}{2} \operatorname{Tr}\left\{h^{T}(x) \frac{\partial^{2} V}{\partial x^{2}} h(x)\right\}\right.\right. \\
& \left.\left.\quad-b(x)\left(v-\frac{1}{2} R^{-1}(x) b^{T}(x)\right)+v^{T} R(x) v\right) d t\right\} \\
& =E\left\{\int_{0}^{T_{0}}-\frac{\partial V}{\partial x} f(x)-\frac{1}{2} \operatorname{Tr}\left\{h^{T}(x) \frac{\partial^{2} V}{\partial x^{2}} h(x)\right\}\right. \\
& =E\left\{-\int_{0}^{T_{0}} \mathscr{L} V(x(\tau)) d \tau+\int_{0}^{T} R(x) v d t\right\} \\
& =E\left(V\left(x_{0}\right)\right)-\lim _{t \rightarrow T_{0}^{-}} E(V(x(t)))+E \int_{0}^{T_{0}} v^{T} R(x) v d t \\
& =E\left(V\left(x_{0}\right)\right)+E \int_{0}^{T_{0}} v^{T} R(x) v d t .
\end{aligned}
$$

Taking $v=0$, that is, $u=u^{*}$, we have

$$
\begin{gathered}
\arg \min _{u} J\left(u, x, x_{0}, w\right)=u^{*}, \\
\min _{u} J\left(u, x, x_{0}, w\right)=E\left(V\left(x_{0}\right)\right) .
\end{gathered}
$$

Corollary 8. Suppose that the conditions of Theorem 6 hold. Then the control law (8) is finite time inverse optimal stabilization in probability for system (1) by minimizing the cost functional (14) with $\lambda>0$ and $a(x), b(x)$ being given as (7) and

$$
\begin{aligned}
& R^{-1}(x)= \begin{cases}\frac{2\left(a(x)+\sqrt{a^{2}(x)+\|b(x)\|^{4}}\right)}{\|b(x)\|^{2}}, & b(x) \neq 0, \\
2 \lambda, & b(x)=0,\end{cases} \\
& l(x)=-\frac{\partial V}{\partial x} f(x)-\frac{1}{2} \operatorname{Tr}\left\{h^{T}(x) \frac{\partial^{2} V}{\partial x^{2}} h(x)\right\} \\
&+\frac{1}{4} R^{-1}(x)\left\|\frac{\partial V}{\partial x} g(x)\right\|^{2} .
\end{aligned}
$$

And the settling time function $T_{0}\left(x_{0}, w\right)$ satisfies $E\left[T_{0}\left(x_{0}\right.\right.$, $w)] \leq\left(V\left(x_{0}\right)\right)^{1-\alpha} / c(1-\alpha), c>0,0<\alpha<1$.

Proof. Using the arguments as Theorem 7, one can prove it.

\section{Simulation Example}

Some designs of finite time stabilizing control laws employ cancellation and do not have satisfactory stability margins, let alone optimality properties. The inverse optimal approach is a constructive alternative to such designs, which achieves desired stability margins. Let us clarify this important issue by an example in this section.

Example 1. Consider the following first-order stochastic nonlinear system:

$$
d x=\left(x^{2 / 3}+u\right) d t+x^{2 / 3} d w .
$$

One possible finite time stabilization design is to let $u$ cancel $x^{2 / 3}$ and add a finite time stabilizing term. This is accomplished with the following control law $u_{1}=-x^{2 / 3}-x^{1 / 3}$ which results in what appears to be a desirable closed-loop system:

$$
d x=-x^{1 / 3} d t+x^{2 / 3} d w .
$$

It is easy to verify that the equilibrium $x=0$ of system (33) is globally finite time stable in probability. However, because of the cancellation, this feedback control law does not have any stability margin: with a slightly perturbed feedback control law $(1+\epsilon) u_{1}(x)$, the closed-loop system

$$
d x=-\left(\epsilon x^{2 / 3}+(1+\epsilon) x^{1 / 3}\right) d t+x^{2 / 3} d w
$$

has solutions which escape to infinity in finite time for any $\epsilon \neq 0$. 


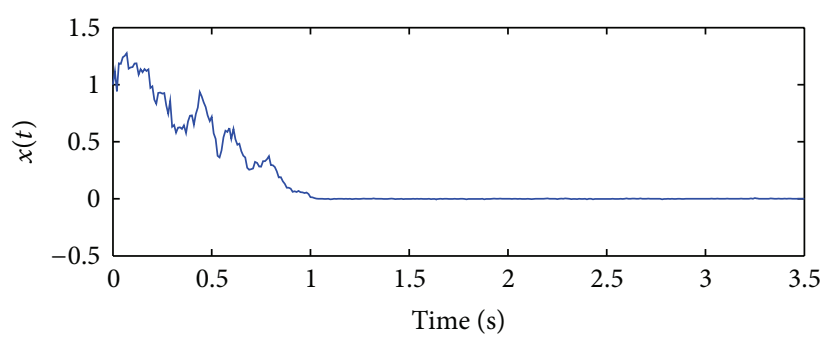

(a)

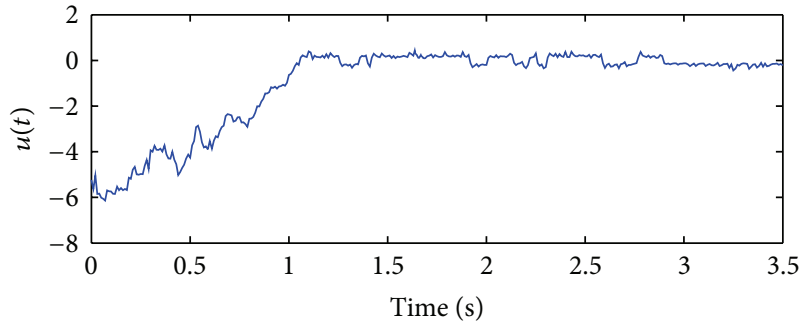

(b)

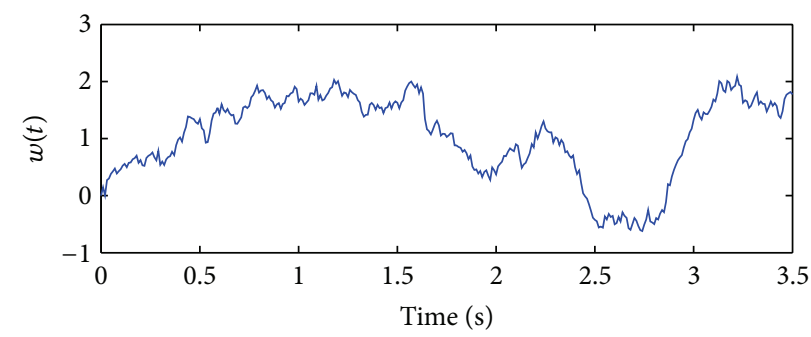

(c)

FIGURE 1: The response of the state $x(t)$, the control $u(t)$, and the Wiener process $w(t)$ in Example 1 for initial conditions as $x(0)=1$.

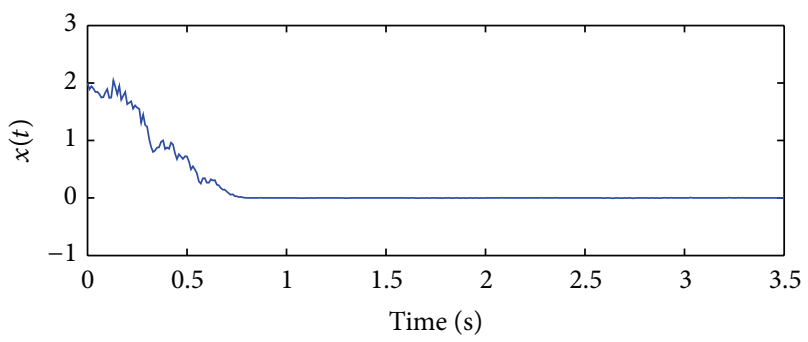

(a)

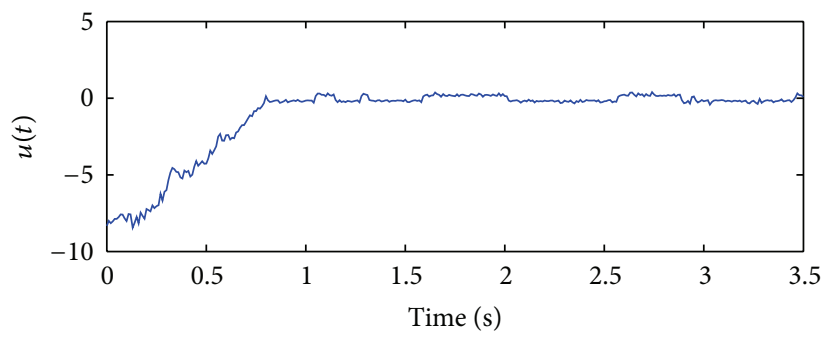

(b)

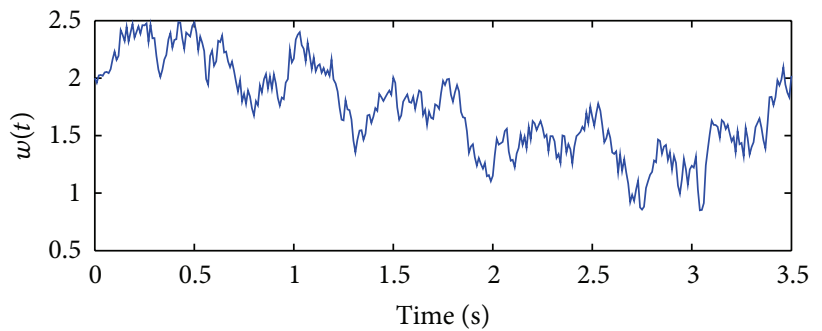

(c)

FIGURE 2: The response of the state $x(t)$, the control $u(t)$, and the Wiener process $w(t)$ in Example 1 for initial conditions as $x(0)=2$.

Let us consider finite time inverse optimal stabilization design for system (32). Let $V(x)=(1 / 2) x^{2}$. It can be computed as

$$
\begin{gathered}
\frac{\partial V(x)}{\partial x} f(x)=x^{5 / 3}, \\
\frac{\partial V(x)}{\partial x} g(x)=x, \\
\frac{1}{2} \operatorname{Tr}\left\{h^{T}(x) \frac{\partial^{2} V}{\partial x^{2}} h(x)\right\}=\frac{1}{2} x^{4 / 3} .
\end{gathered}
$$

One can deduce that $V(x)=(1 / 2) x^{2}$ is an SFT-CLF for system (32). By Theorem 7, we have

$$
\begin{aligned}
u= & k(x)=-x-x^{2 / 3}-x^{1 / 3} \\
& -\operatorname{sgn}(x) \sqrt{\left(x^{2 / 3}+x^{1 / 3}\right)^{2}+x^{2}} .
\end{aligned}
$$

It can be verified that the closed-loop system (32) and (36) satisfies the conditions of Lemma 4 . Thus it has the unique global solution in forward time for all initial conditions.

By Theorem 7, the control law (36) is such that the equilibrium $x=0$ of the closed-loop system (32) and (36) 


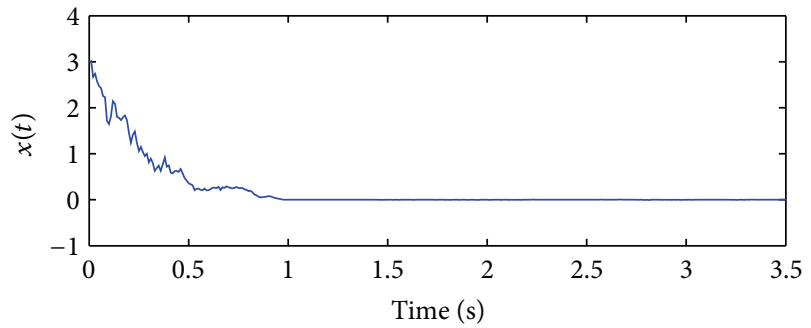

(a)

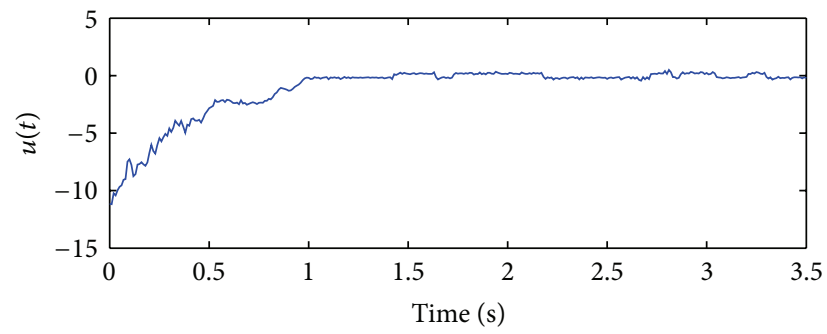

(b)

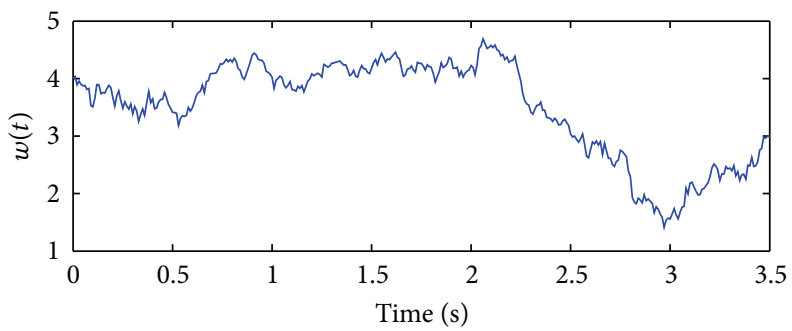

(c)

FIgURE 3: The response of the state $x(t)$, the control $u(t)$, and the Wiener process $w(t)$ in Example 1 for initial conditions as $x(0)=3$.

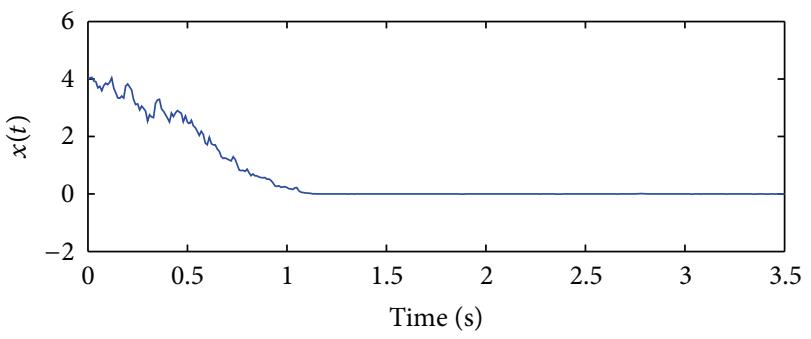

(a)

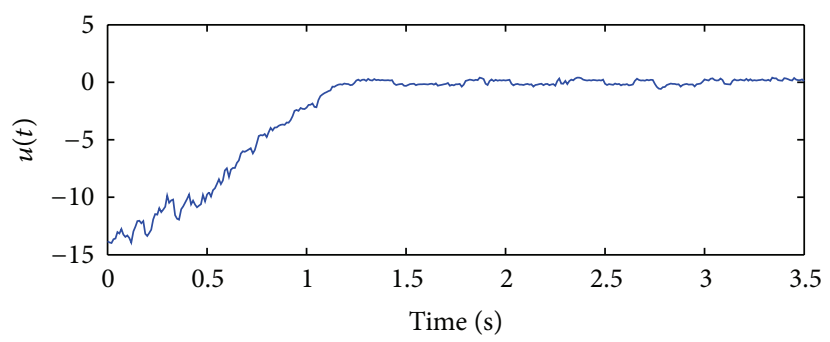

(b)

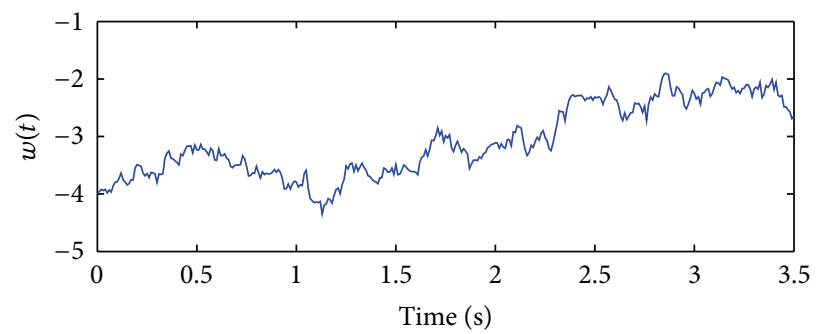

(c)

FIGURE 4: The response of the state $x(t)$, the control $u(t)$, and the Wiener process $w(t)$ in Example 1 for initial conditions as $x(0)=4$.

is finite time stable in probability. And the control law (36) minimizes the cost functional (14) with

$$
l(x)=-\frac{1}{2} x^{5 / 3}+\frac{1}{2} x^{2}+\sqrt{\left(x^{5 / 3}+x^{4 / 3}\right)^{2}+x^{4}},
$$

\section{$R(x)$}

$$
= \begin{cases}\frac{x^{2}}{2 x^{2}+2 x^{5 / 3}+2 x^{4 / 3}+2 \sqrt{\left(x^{5 / 3}+x^{4 / 3}\right)^{2}+x^{4}}}, & x \neq 0, \\ \frac{1}{2}, & x=0 .\end{cases}
$$

Moreover, the control law (36) has two desirable properties.

(i) For $x<0$, it recognizes the beneficial effect of the nonlinearity $x^{2 / 3}$ to enhance the negativity of $\mathscr{L} \mathrm{V}$.

(ii) Instead of cancelling the destabilizing term $x^{2 / 3}$ for $x>0$, the inverse optimal control (36) dominates it and, by doing so, achieves a stability margin.

Figures 1, 2, 3, and 4 show the state $x(t)$ under state feedback control law (36) and the control law $u$ for the initial state $x(0)=1,2,3,4$, respectively, and the response curve of the Wiener process $w(t)$ in the closed-loop system. One can observe that the stabilization is achieved. 


\section{Conclusion}

In this paper, we study the finite time inverse optimal stabilization for stochastic nonlinear systems. First, a concept of the SFT-CLF is presented. Secondly, a control law for finite time stabilization for the closed-loop system is obtained. Furthermore, a sufficient condition is developed for finite time inverse optimal stabilization in probability, and a control law is designed to ensure that the equilibrium of the closed-loop system is finite time inverse optimal stable. Finally, a simulation result shows the effectiveness of the method.

\section{Acknowledgments}

The authors thank the anonymous reviewers for their many helpful suggestions. The authors are grateful for the support of the National Natural Science Foundation of China (Grant nos. 61074011 and 60774011).

\section{References}

[1] V. T. Haimo, "Finite time controllers," SIAM Journal on Control and Optimization, vol. 24, no. 4, pp. 760-770, 1986.

[2] S. P. Bhat and D. S. Bernstein, "Finite-time stability of continuous autonomous systems," SIAM Journal on Control and Optimization, vol. 38, no. 3, pp. 751-766, 2000.

[3] S. P. Bhat and D. S. Bernstein, "Finite-time stability of homogeneous systems," in Proceedings of the American Control Conference, pp. 2513-2514, Albuquerque, NM, USA, 1997.

[4] Y. Orlov, "Finite time stability and robust control synthesis of uncertain switched systems," SIAM Journal on Control and Optimization, vol. 43, pp. 751-766, 2005.

[5] E. Moulay, M. Dambrine, N. Yeganefar, and W. Perruquetti, "Finite-time stability and stabilization of time-delay systems," Systems \& Control Letters, vol. 57, no. 7, pp. 561-566, 2008.

[6] S. G. Nersesov, C. Nataraj, and J. M. Avis, "Design of finite-time stabilizing controllers for nonlinear dynamical systems," International Journal of Robust and Nonlinear Control, vol. 19, no. 8, pp. 900-918, 2009.

[7] S. P. Bhat and D. S. Bernstein, "Continuous finite-time stabilization of the translational and rotational double integrators," IEEE Transactions on Automatic Control, vol. 43, no. 5, pp. 678-682, 1998.

[8] Y. Hong, J. Huang, and Y. Xu, "On an output feedback finitetime stabilization problem," IEEE Transactions on Automatic Control, vol. 46, no. 2, pp. 305-309, 2001.

[9] S. Li, H. Du, and X. Lin, "Finite-time consensus algorithm for multi-agent systems with double-integrator dynamics," Automatica, vol. 47, no. 8, pp. 1706-1712, 2011.

[10] H. Du, S. Li, and C. Qian, "Finite-time attitude tracking control of spacecraft with application to attitude synchronization," IEEE Transactions on Automatic Control, vol. 56, no. 11, pp. 2711-2717, 2011.

[11] H. B. Du and S. H. Li, "Finite-time attitude stabilization for a spacecraft using homogeneous method," Journal of Guidance, Control, and Dynamics, vol. 35, pp. 740-748, 2012.

[12] E. Moulay and W. Perruquetti, "Finite time stability and stabilization of a class of continuous systems," Journal of Mathematical Analysis and Applications, vol. 323, no. 2, pp. 1430-1443, 2006.

[13] Z. Artstein, "Stabilization with relaxed controls," Nonlinear Analysis, vol. 7, no. 11, pp. 1163-1173, 1983.
[14] E. D. Sontag, "A Lyapunov-like characterization of asymptotic controllability," SIAM Journal on Control and Optimization, vol. 21, no. 3, pp. 462-471, 1983.

[15] E. D. Sontag, "A “universal” construction of Artstein's theorem on nonlinear stabilization," Systems \& Control Letters, vol. 13, no. 2, pp. 117-123, 1989.

[16] P. Florchinger, "A universal formula for the stabilization of control stochastic differential equations," Stochastic Analysis and Applications, vol. 11, no. 2, pp. 155-162, 1993.

[17] R. Chabour and M. Oumoun, "On a universal formula for the stabilization of control stochastic nonlinear systems," Stochastic Analysis and Applications, vol. 17, no. 3, pp. 359-368, 1999.

[18] W. Chen and L. C. Jiao, "Finite-time stability theorem of stochastic nonlinear systems," Automatica, vol. 46, no. 12, pp. 2105-2108, 2010.

[19] W. Chen and L. C. Jiao, "Authors' reply to "Comments on 'Finite-time stability theorem of stochastic nonlinear systems," Automatica, vol. 46, pp. 2105-2108, 2010.

[20] W. Chen and L. C. Jiao, "Authors' reply to "Comments on 'Finite-time stability theorem of stochastic nonlinear systems"', Automatica, vol. 47, no. 7, pp. 1544-1545, 2011.

[21] R. A. Freeman and P. V. Kokotovic, "Inverse optimality in robust stabilization," SIAM Journal on Control and Optimization, vol. 34, no. 4, pp. 1365-1391, 1996.

[22] R. Situ, Theory of Stochastic Differential Equations with Jumps and Applications, Mathematical and Analytical Techniques with Applications to Engineering, Springer, New York, NY, USA, 2005.

[23] X. Cai, L. Liu, J. Huang, and W. Zhang, "Globally asymptotical stabilisation for a class of feedback linearisable differential inclusion systems," IET Control Theory \& Applications, vol. 5, no. 14, pp. 1586-1596, 2011.

[24] P. Chen, C. Z. Hou, and Y. Feng, Stochastic Mathematics, National Defence Industry Press, Beijing, China, 2008. 


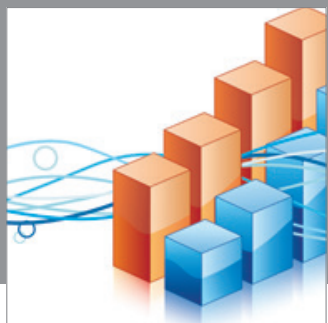

Advances in

Operations Research

mansans

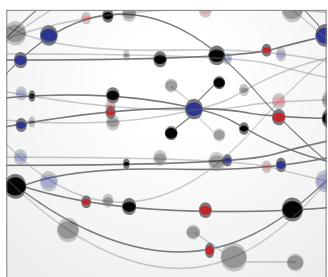

The Scientific World Journal
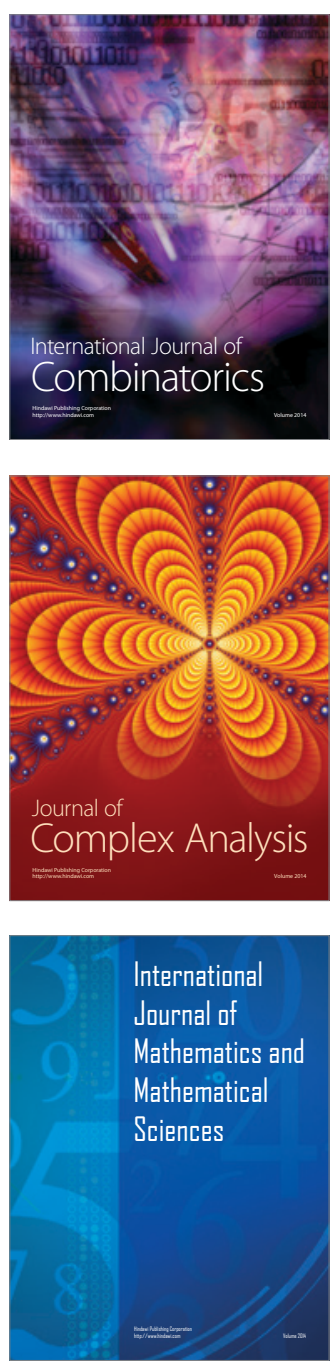
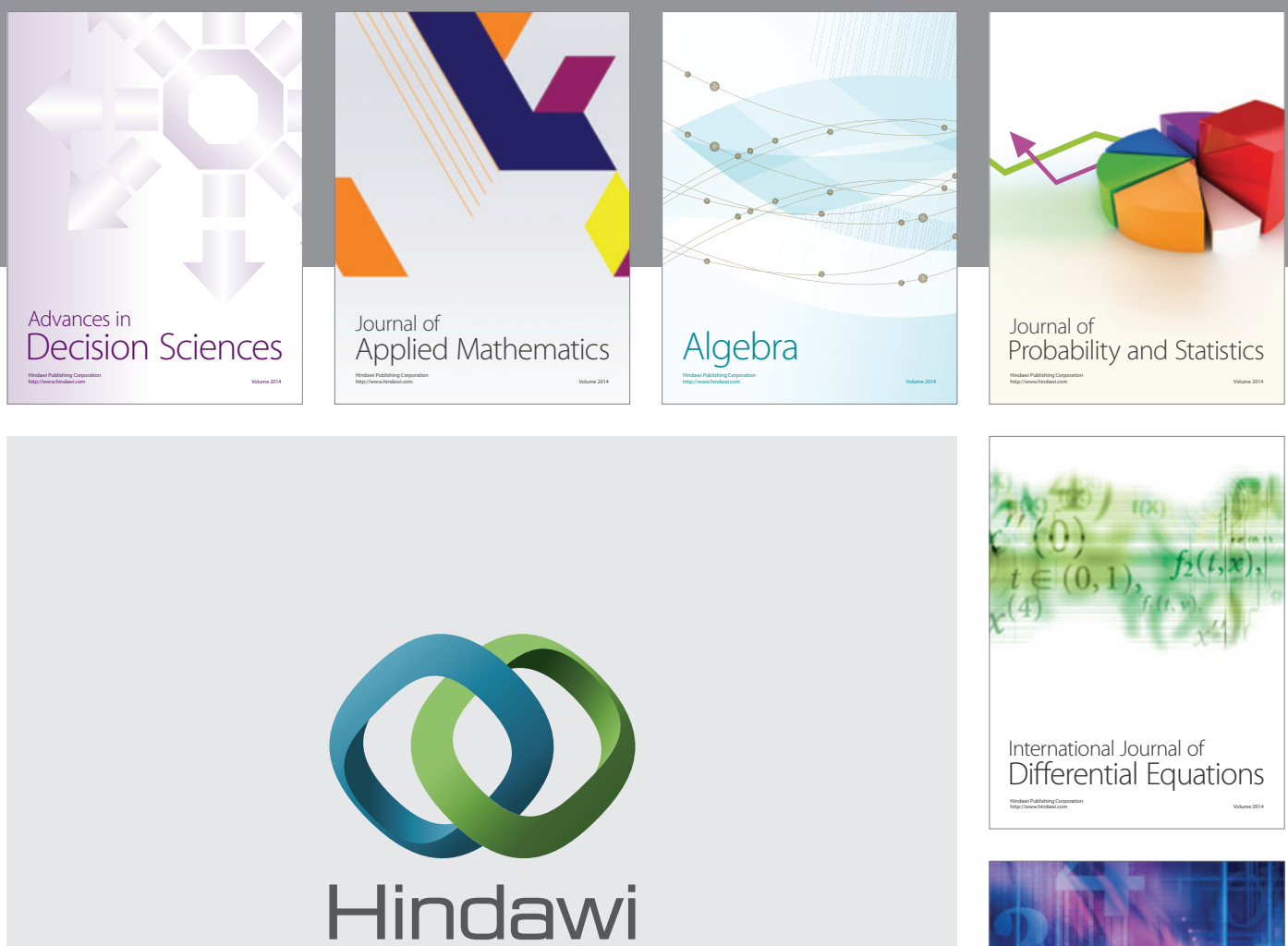

Submit your manuscripts at http://www.hindawi.com
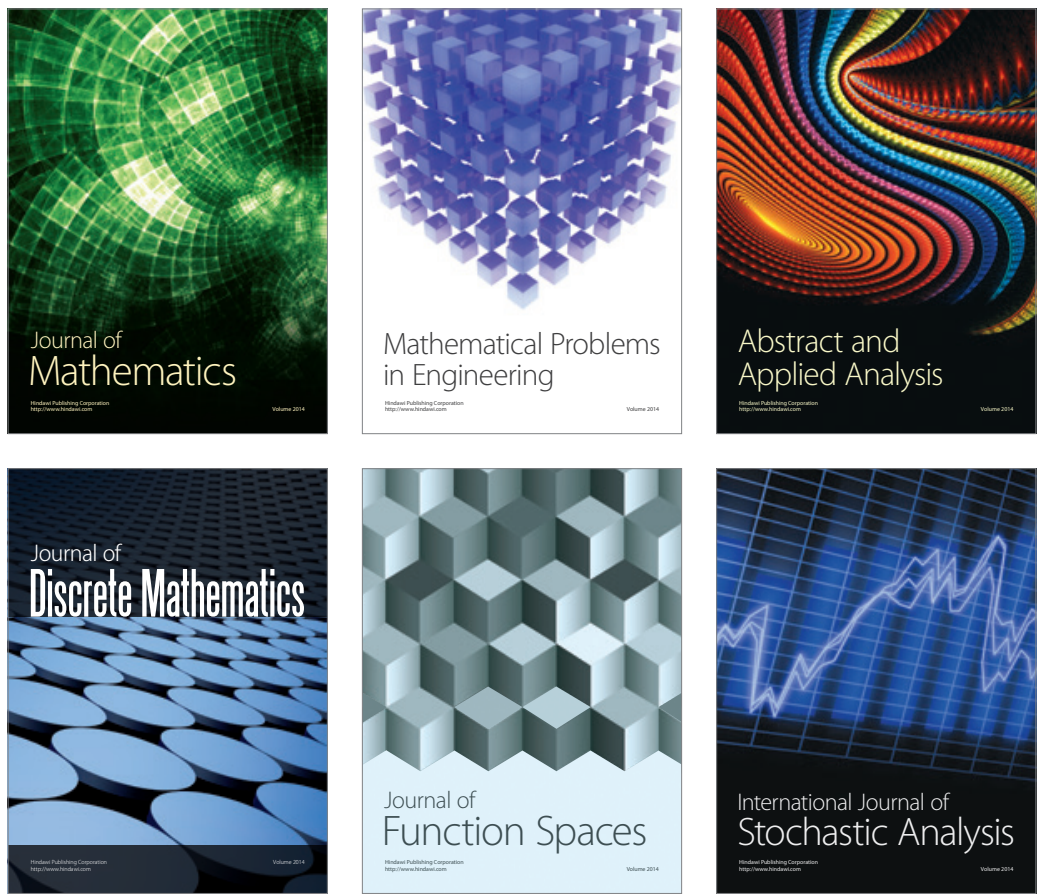

Journal of

Function Spaces

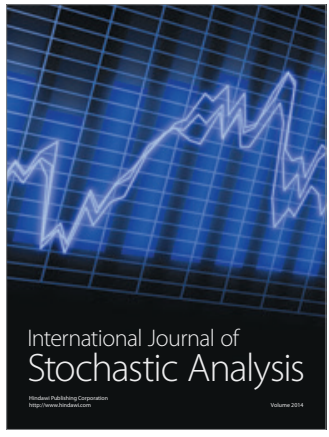

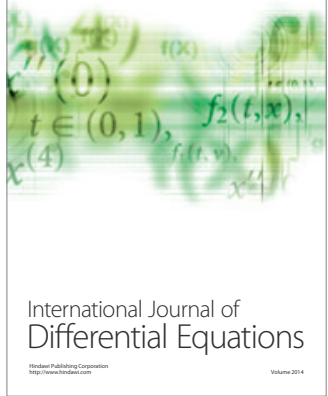
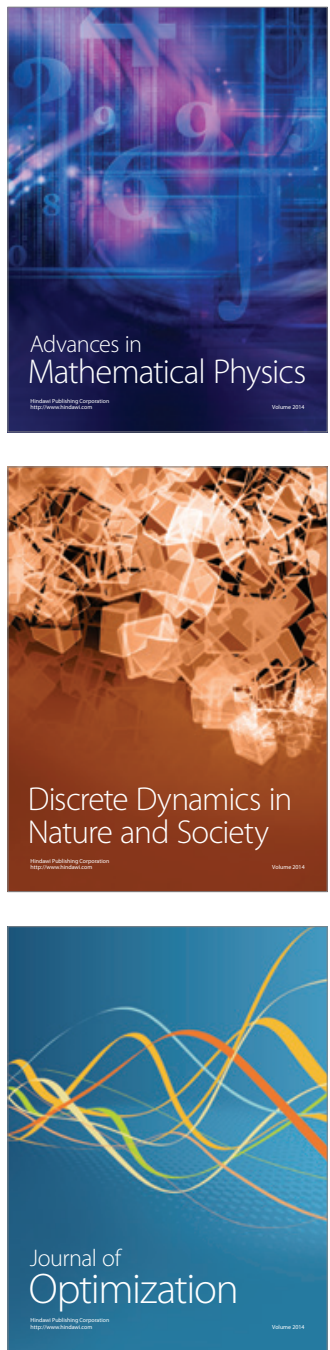